\title{
Theoretical Analysis and Optimum Design of High Speed Air Film Thrust Bearings (Application to Optimum Design Problem)"
}

\author{
Hiromu HASHIMOTO $^{* *}$, Masayuki OCHIAI ${ }^{* *}$ and Tadashi NANBA ${ }^{* * *}$ \\ ${ }^{* *}$ Department of Mechanical Engineering, Tokai University \\ 1117 Kitakaname Hiratsuka-shi Kanagawa Japan \\ E-mail: hiromu@keyaki.cc.u-tokai.ac.jp \\ ${ }^{* * *}$ Graduate School of Engineering, Tokai University
}

\begin{abstract}
Hydrodynamic air film thrust bearings are widely used for very high speed, lightly loaded rotating machinery such as gas expander, compressor, gyroscope and business machines, etc. In the design of hydrodynamic air film thrust bearings, it is of cardinal importance to enhance the friction and stability capacities of air films for keeping the minimum friction loss within a particular level and for minimizing the vibration due to external excitations. Among various types of hydrodynamic air film thrust bearings, spiral and herring bone types of grooved bearings have an advantage of high stability and load carrying capacity, but the characteristics of the bearings depend on many design parameters. Therefore, when these parameters are designed suitably, it is expected to improve considerably the friction and stability characteristics of the bearings. In this paper, the optimum design methodology is presented to minimize the friction torque and also to maximize the stiffness of air film for spiral and herring bone types of grooved air film thrust bearings, and the applicability of the methodology is verified experimentally.
\end{abstract}

Key words: Machine Element, Thrust Air Bearings, Numerical Analysis, Optimum Design, Measurement, Static Characteristics, Dynamic Characteristics

\section{Introduction}

Hydrodynamic air film thrust bearings are very important machine elements to support lightly loaded, high speed rotating machinery such as gas expander, compressor, micro gas turbine, color copier etc. Among various types of air film thrust bearings, spiral and herring bone types of grooved bearings are mainly used. However, it is not so easy to design such types of grooved bearings due to their complex groove geometries. Nevertheless, the literatures treating the effective design methodology of the bearings are very limited as compared with the literatures on the bearing characteristic analysis ${ }^{(1) \sim(8)}$.

In the previous paper ${ }^{(9)}$, the authors presented the experimental method to measure the static and dynamic characteristics of various types of air film thrust bearings and verified the applicability of the numerical analysis method combining DF method and Newton-Raphson iteration method based on the boundary fitted coordinate, by comparing the calculated results with the measured data for four types of air film thrust bearings.

In this subsequent paper, the optimum design methodology to enhance the quality of spiral and herring bone types of grooved bearings based on the numerical analysis method verified in the previous paper is described. The optimum design variables to minimize the ratio of friction torque to spring coefficient of air film are determined by the hybrid optimization method combining the successive quadratic programming (SQP) and the direct search method ${ }^{(10)}$. The predicted results of friction torque and spring coefficient are compared with the measured data and the applicability of the present methodology is verified experimentally. 


\section{Nomenclature}

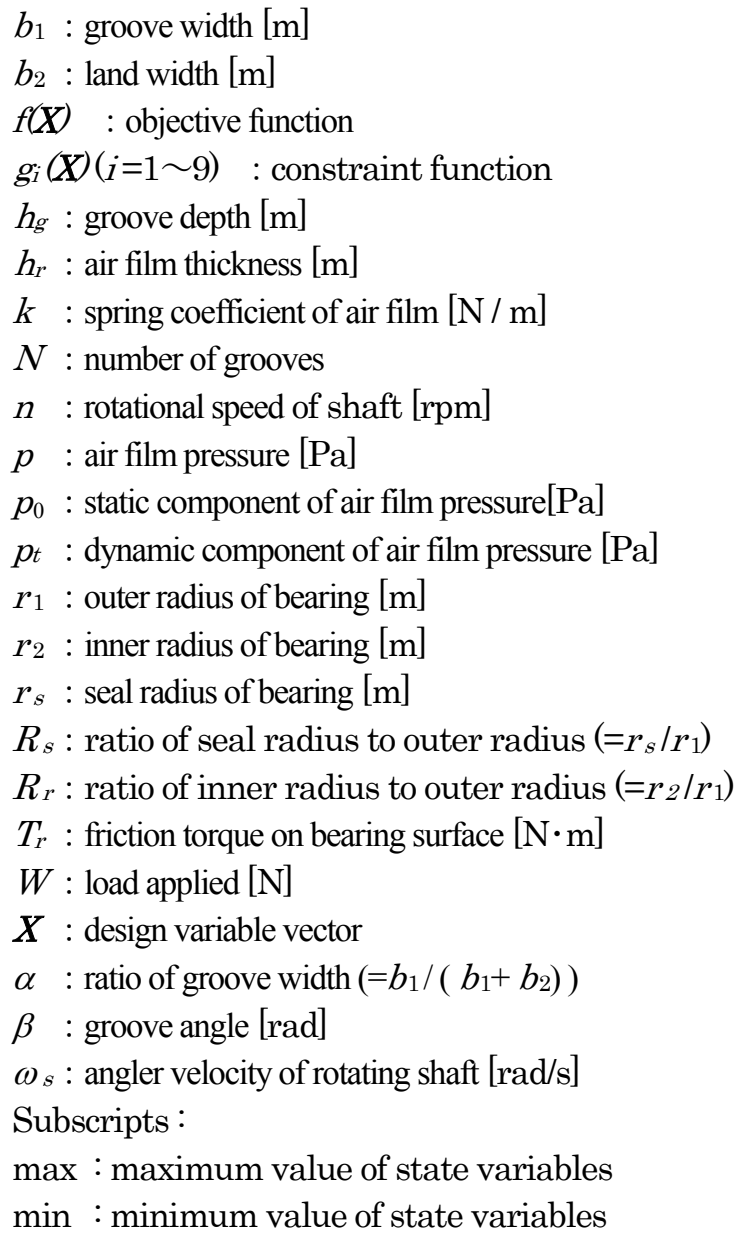

\section{Formulation of Optimum Design Problems}

In this paper, the optimum design problems are defined to maximize spring coefficient of air film and minimize friction torque under maintaining the allowable air film thickness. The allowable air film thickness is very important factor to be considered, which is one of the constraint conditions to keep the safety operation avoiding the surface contact and seizure. The friction torque on the bearing surface should be reduced from the point of view of saving the energy consumption. On the other hand, the spring coefficient of air film should be increased to improve the dynamic response for the external excitation. In the optimum design, the damping coefficient is neglected as compared with the spring coefficient because the effect of damping coefficient on the dynamic response is much smaller than that of spring coefficient as shown in the previous paper ${ }^{(9)}$.

Figure 1 shows the geometry of spiral and herring bone types of grooved bearings to be treated in this paper. In the formulation of optimum design problems, the outer and inner radii of bearing $r_{1}, r_{2}$, load applied to the bearing $W$, and the rotational shaft speed $n$, are given as the prescribed values.

The design variable vector $X$ is defined as follows.

$$
X=\left(h_{g}, R_{s}, \alpha, \beta, N\right)
$$

The objective function to minimize the friction torque and maximize the spring coefficient of air film simultaneously is defined as follows.

$$
f(X)=T_{r} / k
$$




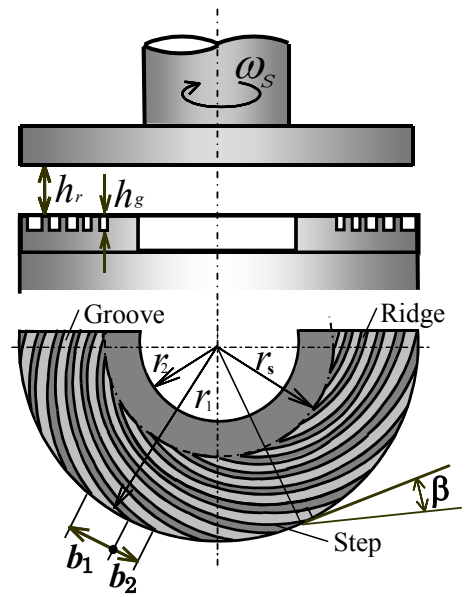

(a) Spiral grooved bearing

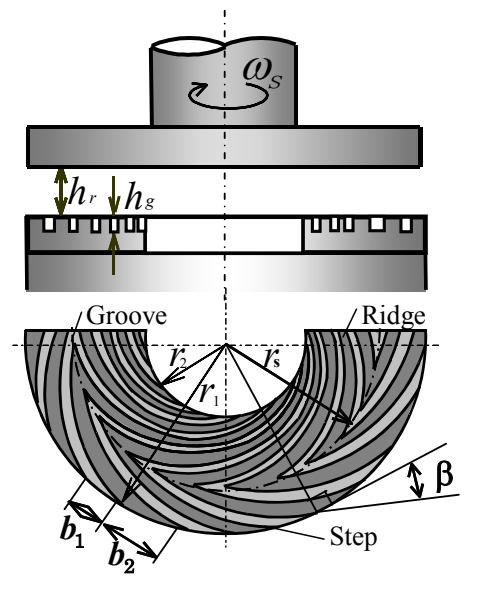

(b) Herring bone grooved bearing

Fig.1 Geometries of four types of air film thrust bearings

The constraint conditions for design variables and state variables are expressed as follows.

$$
g_{i}(X) \geq 0(i=1 \sim 9)
$$

where, the constraint functions $g_{i}(i=1 \sim 9)$ are given as follows.

$$
\left.\begin{array}{l}
g_{1}=h_{g}-h_{g \min }, g_{2}=h_{g \max }-h_{g}, \\
g_{3}=\beta-\beta_{\min }, g_{4}=\beta_{\text {max }}-\beta, \\
g_{5}=R_{s}-R_{s \min }, g_{6}=R_{s \max }-R_{s}, \\
g_{7}=\alpha-\alpha_{\min }, g_{8}=\alpha_{\max }-\alpha, \\
g_{9}=h_{r}-h_{r \min }
\end{array}\right\}
$$

The friction torque, $T_{r}$, and spring coefficient of air film, $k$, are obtained by the numerical analysis method developed in the previous paper ${ }^{(9)}$. The outline of the numerical analysis method is briefly described as follows.

In the spiral and herring bone types of grooved bearings, the geometry of boundary between groove and land is very complicated. To analyze such complicated bearings, the groove geometries are transformed to simple combination of straight lines and circles based on the boundary fitted coordinate system.

From the mass flow rate balance in the control volume, the following type of equivalent Reynolds equation is obtained.

$$
\begin{aligned}
& Q^{\xi}{ }_{2 I}+Q^{\xi}{ }_{1 I I I}-Q^{\xi}{ }_{2 I I}-Q^{\xi}{ }_{2 I V} \\
& +Q^{\eta}{ }_{2 I}+Q^{\eta}{ }_{1 I I}-Q^{\eta}{ }_{2 I I I}-Q^{\eta}{ }_{1 I V}=Q^{V}
\end{aligned}
$$

where, the mass flow rate $Q^{\xi}, Q^{\eta}$, and $Q^{v}$ are given, respectively, as follows.

$$
\begin{aligned}
& Q^{\xi}=\int_{\eta_{1}}^{\eta_{2}} \rho\left(-A \frac{\partial p}{\partial \xi}+B \frac{\partial p}{\partial \eta}+D+E\right) d \eta \\
& Q^{\eta}=\int_{\xi_{1}}^{\xi_{2}} \rho\left(B \frac{\partial p}{\partial \xi}-C \frac{\partial p}{\partial \eta}+F+G\right) d \xi \\
& Q^{v}=\int_{\xi_{1}}^{\xi_{2}} \int_{\eta_{1}}^{\eta_{2}} \frac{\partial(\rho h)}{\partial t}|J| d \eta d \xi
\end{aligned}
$$

where: 


$$
\begin{aligned}
& A=a \frac{h^{3}}{12 \mu J}, B=b \frac{h^{3}}{12 \mu J}, C=c \frac{h^{3}}{12 \mu J}, D=-\frac{r \omega h}{2} r_{\eta} \text {, } \\
& E=\frac{\rho r \omega^{2} h^{3}}{40 \mu} r \theta_{\eta}, F=\frac{r \omega h}{2} r_{\xi}, G=\frac{\rho r \omega^{2} h^{3}}{40 \mu} r \theta_{\xi}, \\
& a=\left(r \theta_{\eta}\right)^{2}+r_{\eta}{ }^{2}, b=\left(r \theta_{\xi}\right)\left(r \theta_{\eta}\right)+r_{\xi} r_{2}, c=\left(r \theta_{\xi}\right)^{2}+r_{\xi}{ }^{2} \\
& J=r_{\xi}\left(r \theta_{\eta}\right)-r_{\eta}\left(r \theta_{\xi}\right), r_{\xi}=\frac{\partial r}{\partial \xi}, r_{\eta}=\frac{\partial r}{\partial \eta}, r \theta_{\xi}=r \frac{\partial \theta}{\partial \xi}, r \theta_{\eta}=r \frac{\partial \theta}{\partial \eta}
\end{aligned}
$$

In Eq.(5), the subscripts 1, 2 and I $\sim$ IV refer to the divided region in the control volume.

Assuming a small vibration of bearing gap in the axial direction, the air film thickness and pressure are expressed, respectively, as follows.

$$
\left.\begin{array}{l}
h=h_{0}+\varepsilon e^{j \omega_{f} t} \\
p=p_{0}+\varepsilon p_{t} e^{j \omega_{f} t}
\end{array}\right\}
$$

where the parameter $\varepsilon$ represents the function of air film thickness and the bound of $\varepsilon$ is approximately within \pm 10 percent of the steady state air film thickness. The subscripts 0 and $t$ mean the physical quantities under the steady and unsteady operation conditions, respectively.

Substituting Eq.(8) into Eq.(5) and separating the results according to the order of the small parameter $\varepsilon$, the following two sets of equations are obtained for the 0 -th and the 1 -st orders of parameter $\varepsilon$.

$$
\begin{aligned}
F_{0}\left(p_{0}\right)= & Q^{\xi}{ }_{2 I 0}+Q^{\xi}{ }_{1 I I I 0}-Q^{\xi}{ }_{2 I I 0}-Q^{\xi}{ }_{1 I V 0} \\
+ & Q^{\eta}{ }_{2 I 0}+Q^{\eta}{ }_{1 I I 0}-Q^{\eta}{ }_{2 I I I 0}-Q^{\eta}{ }_{1 I V 0}=0 \\
F_{t}\left(p_{t}, p_{0}\right) & =Q^{\xi}{ }_{2 I t}+Q^{\xi}{ }_{1 I I I t}-Q^{\xi}{ }_{2 I I t}-Q^{\xi}{ }_{1 I V t} \\
& +Q^{\eta}{ }_{2 I t}+Q^{\eta}{ }_{1 I I t}-Q^{\eta}{ }_{2 I I I t}-Q^{\eta}{ }_{1 I V t}-Q^{v}{ }_{t}=0
\end{aligned}
$$

Descretizing Eqs.(9.a) and (9.b) by the finite difference method and solving them in turn by the Newton-Raphson iteration method, the static and dynamic pressure components, $p_{0}$ and $p_{t}$, are obtained. After obtaining these pressure components, the friction torque and spring coefficient are given, respectively, by the following integrations.

$$
\begin{aligned}
& T_{r}=\int_{r_{1}}^{r_{2}} \int_{0}^{2 \pi}\left(-\frac{r h_{0}}{2} \frac{\partial p_{0}}{\partial \theta}+\frac{\mu r^{3} \omega_{s}}{h_{0}}\right) d r d \theta \\
& k=\int_{r_{1}}^{r_{2}} \int_{0}^{2 \pi} \operatorname{Re}\left(-p_{t}\right) r d r d \theta
\end{aligned}
$$

where the steady state air film thickness,$h_{r 0}$, is calculated from the equilibrium condition between the load applied and the air film reaction force obtained by the integration of $p_{0}$ over the bearing surface.

Based on the above definitions, the optimum design problems for the air film thrust bearings are formulated as follows.

Find $X$ to minimize $f(X)$

$$
\text { subjected to } g_{i}(X) \geq 0(i=1 \sim 9)
$$

Figure 2 shows the flow chart of the optimum design procedure. The procedure consists of three stages. The first stage is the preparation of optimum design. The prescribed values contain the invariable parameters such as the upper and lower limits of each design variable etc. A sensitivity analysis is performed to investigate the effects of each design parameter on the friction torque and spring coefficient. 

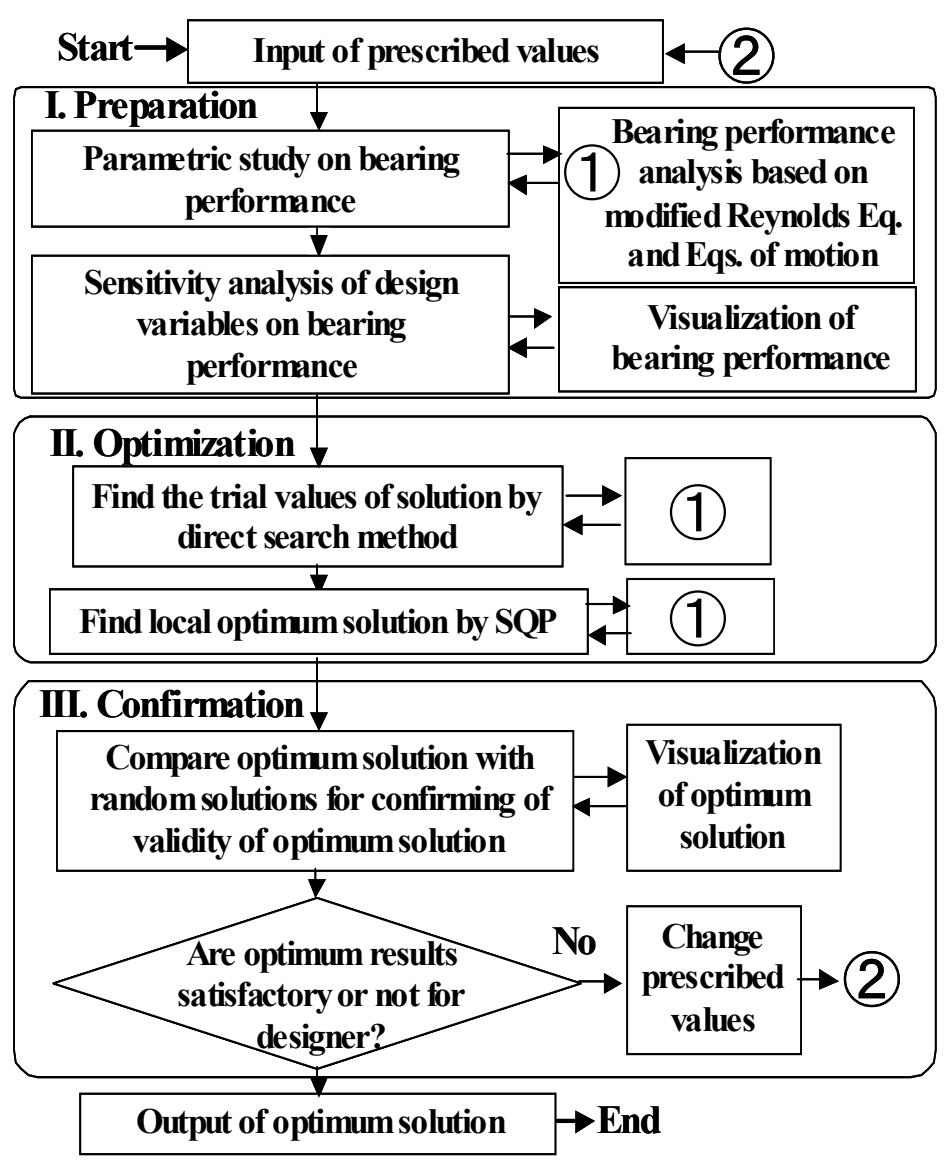

Fig.2 Flow chart for the optimum design methodology

The second stage is the optimization. The hybrid optimization technique combining the direct search method and the successive quadratic programming (SQP) is applied. SQP is a powerful method to obtain the exact local optimum solutions for nonlinear optimization problems. However, it sometimes can not find the global optimum solution when the objective function has the multi peaks. Thus, the direct search method is used first to roughly search for several optimum solution conditions, then, SQP is used to find the local optimum solutions. The global optimum solution is finally obtained by choosing the smallest local solution.

The last stage is the confirmation of the optimum solution. The validity of the optimum solution is confirmed by comparing the value of the objective function calculated from the optimum design variables with those from randomly selected design variables. Whenever the optimum solution is not satisfactory, the total procedure is repeated under the different conditions.

\section{Examples of Optimum Design}

In the optimum design of spiral and herring bone types of grooved air film bearings, the prescribed parameters are given as shown in Table 1.

The number of grooves, $N$, is the integer and the candidates of the optimum value of $N$ are selected as $N=8,12,16,20$ and 24. At first, the optimum values of the objective function are calculated for these fixed values of $N$ based on the procedure as shown in Fig.2.

Figure 3 shows the relation between the optimized objective function and the number of grooves for the fixed value of the rotational shaft speed of $n=40000 \mathrm{rpm}$. As can be seen in Fig.3, in both cases of spiral grooved and herring bone grooved bearings, the optimum 
Table 1 Given variables and constraints

\begin{tabular}{|c|c|}
\hline Parameters & Values \\
\hline Minimum groove depth & $h_{g \min }=10 \mu \mathrm{m}$ \\
\hline Maximum groove depth & $h_{g \max }=\mathbf{3 0} \mu \mathrm{m}$ \\
\hline $\begin{array}{l}\text { Minimum seal radius to outer } \\
\text { radius ratio }\end{array}$ & $R_{s \min }=0.4$ \\
\hline $\begin{array}{l}\text { Maximum seal radius to outer } \\
\text { radius ratio }\end{array}$ & $R_{s \max }=0.9$ \\
\hline Minimum groove width ratio & $\alpha_{\min }=\mathbf{0 . 1}$ \\
\hline Maximum groove width ratio & $\alpha_{\max }=0.9$ \\
\hline Minimum spiral angle & $\beta_{\min }=15 \mathrm{deg}$ \\
\hline Maximum spiral angle & $\beta_{\max }=60 \mathrm{deg}$ \\
\hline Minimum film thickness & $h_{r \min }=5 \mu \mathrm{m}$ \\
\hline Groove number & $N=8,12,16,20,24$ \\
\hline Outer radius & $R_{1}=32 \mathrm{~mm}$ \\
\hline Inner radius to outer radius ratio & $\boldsymbol{R}_{r}=\mathbf{0 . 4}$ \\
\hline Bearing load & $W=14.7 \mathrm{~N}$ \\
\hline Rotational speed & $n=20000 \sim 80000 \mathrm{rpm}$ \\
\hline
\end{tabular}

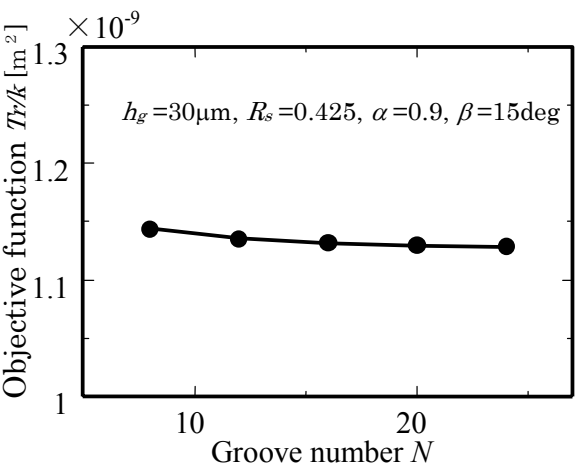

(i) Spiral grooved bearing

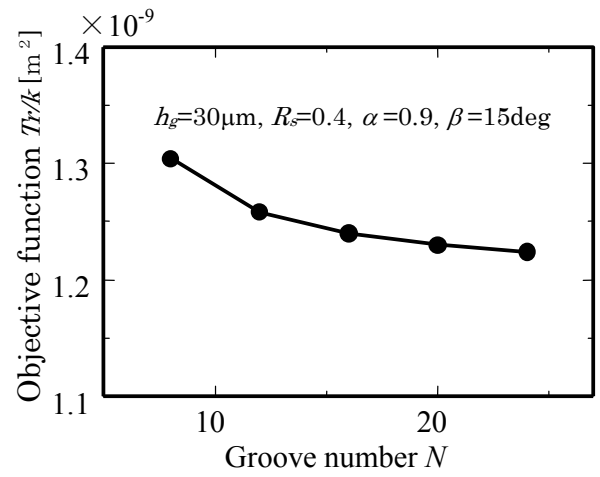

(ii) Herring bone grooved bearing

Fig.3 Optimized design variables

value of the objective function decreases with an increase of the number of groove and approaches to the constant value at $N=24$. As shown in the later, the sensitivity of the rotational shaft speed on the objective function is very small, and then the optimum value of the number of groove is determined as $N=24$.

For the fixed value of $N=24$, the trial values of four design variables, the ratio of seal radius to outer radius $R_{s}$, groove depth $h_{g}$, groove width ratio $\alpha$, and the groove angle $\beta$, are found based on the direct search method. As a result, the trial values of these variables are given as $R_{s}=0.45, h_{g}=30 \mu \mathrm{m}, \alpha=0.9$ and $\beta=15.0 \mathrm{deg}$. Finally, applying SQP for these values, the optimum solutions are obtained.

The optimum design variables of $h_{g}, R_{s}, \alpha$ and $\beta$ for the spiral grooved bearings are shown in Fig.4 as a function of the rotational shaft speed. The optimum values of $h_{g}$ and $\alpha$ become the upper limit of each design variable. On the other hand, the optimum value of $\beta$ 


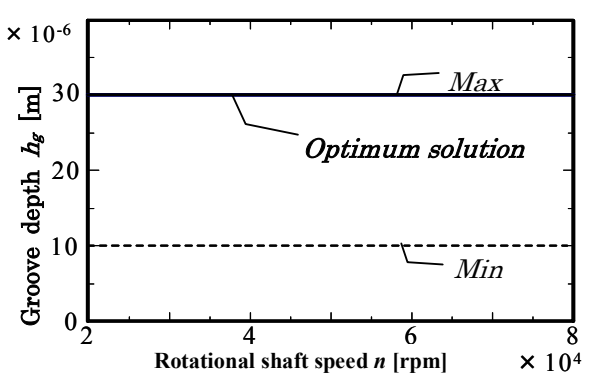

(i) Groove depth $h_{g}$

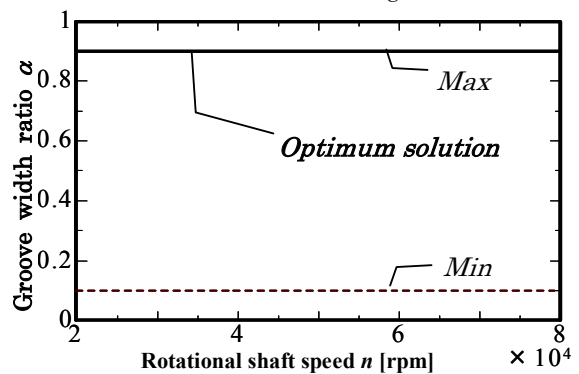

(iii) Groove width ratio $\alpha$

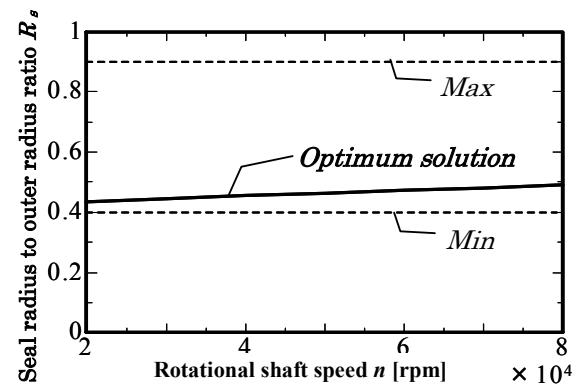

(ii) Seal radius to outer radius ratio $R_{s}$

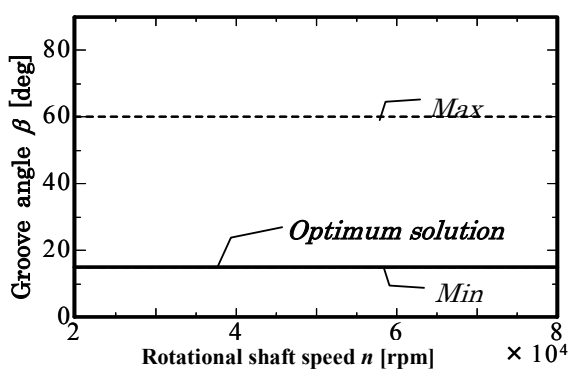

(iv) Groove angle $\beta$ [deg]

Fig.4 Optimum design variables for spiral grooved bearing

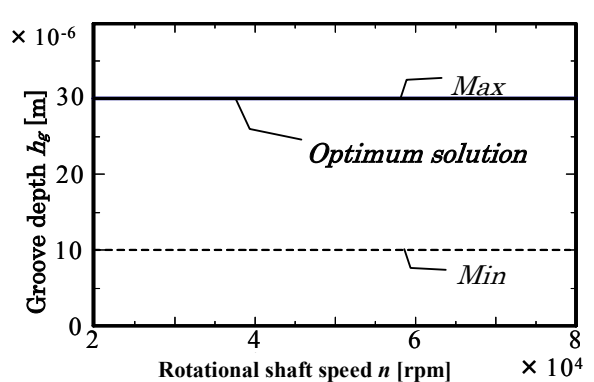

(i) Groove depth $h_{g}$

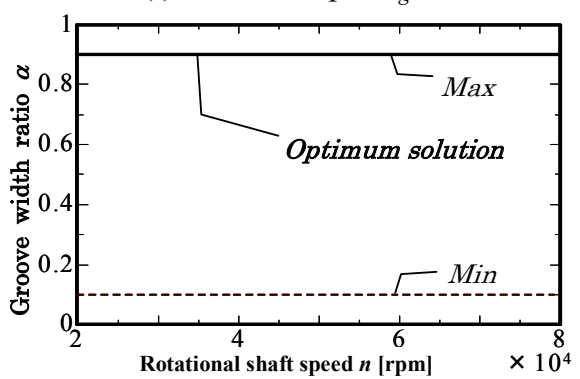

(iii) Groove width ratio $\alpha$

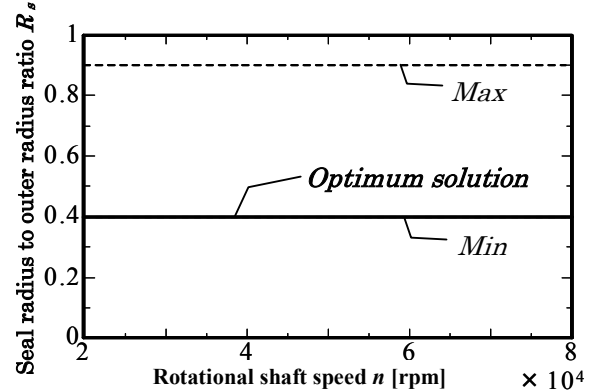

(ii) Seal radius to outer radius ratio $R_{s}$

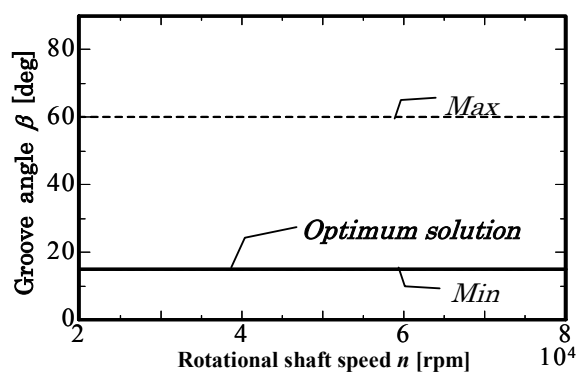

(iv) Groove angle $\beta$ [deg]

Fig.5 Optimum design variables for herring bone grooved bearing

takes the lower limit. The optimum value of $R_{s}$ increases slightly with an increase of the rotational shaft speed.

Figure 5 shows the relation between the optimum design variables and the rotational shaft speed for the herring bone grooved bearings. The quite same results are obtained as the results for the spiral grooved bearings except the ratio of seal radius to outer radius $R_{s}$. In the case of herring bone grooved bearings, the optimum value of $R_{s}$ takes the lower limit.

Figure 6 shows a comparison of the geometries of optimized spiral and herring bone types of grooved bearings with those of the conventional types of bearings designed based on MTI data ${ }^{(11)}$. The optimized spiral grooved bearing has the larger number of grooves $N$, deeper groove depth $h_{g}$, and smaller groove width ratio $\alpha$, and groove angle $\beta$, as compared with the conventional designed bearings.

On the other hand, in the case of the optimized herring bone grooved bearing, there is 


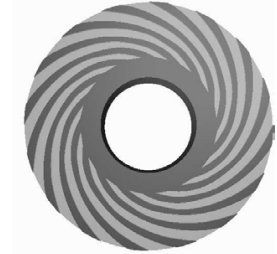

(a-1)Conventional type of spiral grooved $\left(h_{g}=20[\mu \mathrm{m}], R_{s}=0.58\right.$, $\alpha=0.6, \beta=16$ [deg], $N=16$ )
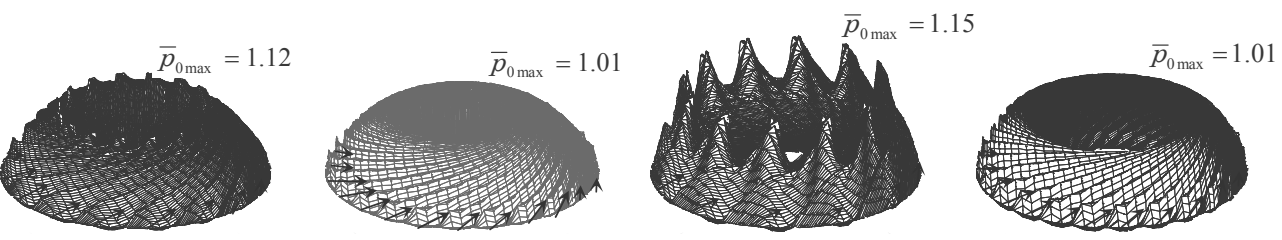

( i ) Static component $\bar{p}_{0 \max }$

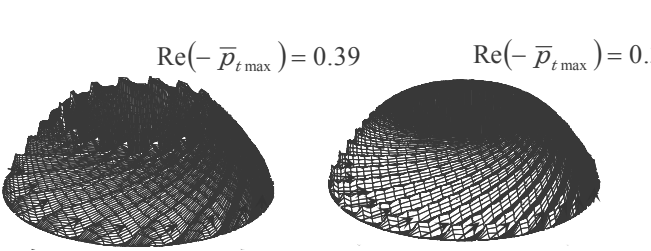

$$
\operatorname{Re}\left(-\bar{p}_{t \max }\right)=0.47
$$
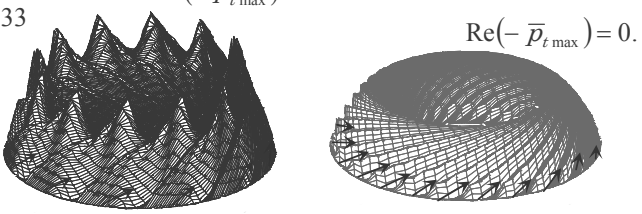

(ii) Real part of dynamic component $\operatorname{Re}\left(-\bar{p}_{t \max }\right)$

(a-1)Conventional type of spiral grooved $\left(h_{g}=20[\mu \mathrm{m}], R_{s}=0.58\right.$ $\alpha=0.6, \beta=16$ [deg], $N=16$ ) (a-2) Optimized spiral grooved

$\left(h_{g}=30[\mu \mathrm{m}], R_{s}=0.425\right.$, $\alpha=0.9, \beta=15$ [deg] $N=24$ ) $(b-1)$ Conventional type of (b-2) herring bone grooved $\left(h_{g}=20[\mu \mathrm{m}], R_{s}=0.76\right.$, $\alpha=0.6, \beta=16$ [deg], $N=12$ )

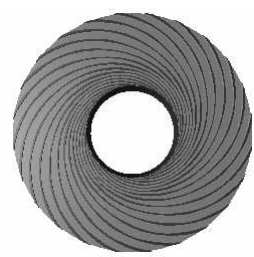

b-2) Optimized herring bone grooved $\left(h_{g}=30[\mu \mathrm{m}], R_{s}=0.4\right.$ $\alpha=0.9, \beta=15$ [deg], $N=24$ )

Fig.7 Static and dynamic normalized pressure distributions $(n=30000[\mathrm{rpm}])$

no seal radius on the grooves and the groove geometry becomes essentially the same as the spiral grooved bearing.

Figure 7 shows the static pressure components and the real parts of dynamic pressure components for the conventional and optimized bearings in the cases of spiral and herring bone types of grooved bearings. Integrating the static pressure component $p_{0}$ over the bearing surface, the load carrying capacity (air film reaction force) is obtained. On the other hand, integrating the real part of the dynamic pressure component, the spring coefficient of air film is obtained.

In the case of the optimized spiral grooved bearing, as shown in Fig.6, the number of grooves increases and the width of groove and land decreases. The groove angle becomes smaller and the length of groove becomes larger. Moreover, the seal radius becomes much smaller. As a result, in the optimized spiral grooved bearing, the area of pressure generation becomes wider and the distribution of the pressure tends to be averaged over the bearing surface as compared with the conventional spiral groove bearing. The same tendency can be seen in the static pressure of the optimized herring bone bearing. The distribution of pressure becomes much smooth as compared with case of the conventional herring bone grooved bearing.

The real parts of the dynamic pressure components of both types of bearings are more averaged and distributed more widely over the bearing surface as compared with the cases 
Table.2 Bearing specification

\begin{tabular}{|c|c|c|c|c|c|c|}
\hline Case & Obj. $f(\boldsymbol{X})$ & $h_{s}[\mu \mathrm{m}]$ & $R_{\boldsymbol{s}}$ & $\alpha$ & $\beta[\mathrm{deg}]$ & $N$ \\
\hline Optimum designed & 1.224 & 30 & 0.40 & 0.90 & 15 & 24 \\
\hline Random 1 & 1.688 & 20 & 0.43 & 0.85 & 20 & 16 \\
\hline Random 2 & 1.784 & 18 & 0.72 & 0.80 & 25 & 20 \\
\hline Random 3 & 2.308 & 15 & 0.80 & 0.20 & 30 & 24 \\
\hline Random 4 & 2.865 & 30 & 0.60 & 0.65 & 40 & 8 \\
\hline Random 5 & 1.347 & 28 & 0.65 & 0.88 & 16 & 20 \\
\hline
\end{tabular}

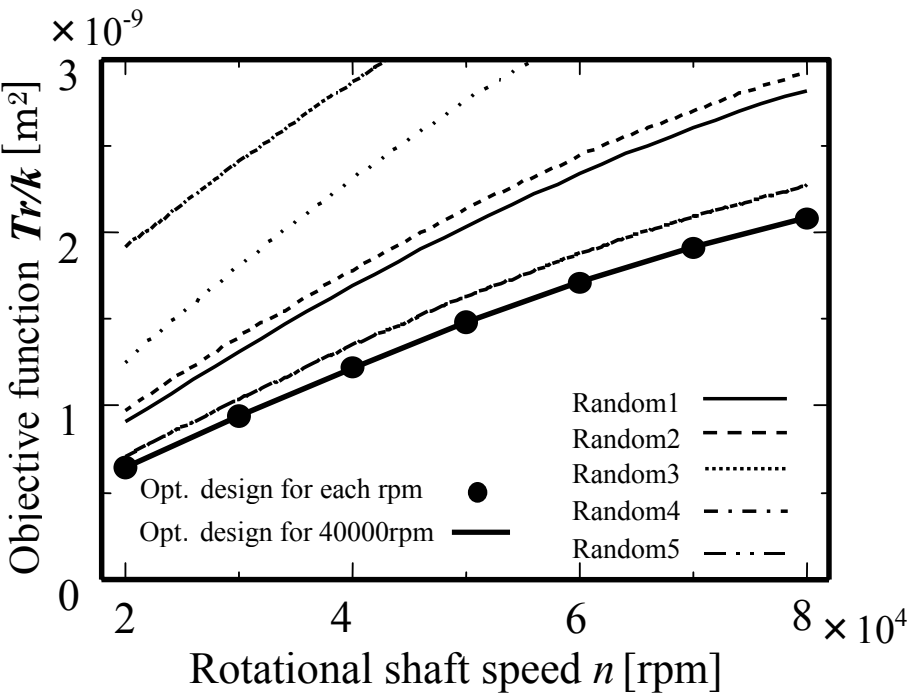

Fig. 8 Comparison between the optimum designed and non-optimum designed herringbone grooved bearing

of conventional bearings.

To confirm the effectiveness of optimum design presented here, the values of design variables and objective function for the optimized herring bone grooved bearing are compared with those for randomly designed herring bone grooved bearings for the fixed rotational shaft speed of $n=40000 \mathrm{rpm}$ in Table 2. Moreover, the variation of objective function is presented in Fig. 8 for a wide range of rotational shaft speed from 20000 to 40000rpm.

As shown in these data, the objective function of the optimized bearing takes the minimum value in all bearings presented for a wide range of rotational shaft speed, and the results for the bearing optimized at $n=40000 \mathrm{rpm}$ coincide with the results for the bearings optimized at each rotational shaft speed, as indicated by the plots in Fig.8. It is confirmed from these data that the sensitivity of the rotational shaft speed on the optimized bearing geometry is negligible small and the optimum design methodology proposed in this paper effectively improve the quality of the air film thrust bearings.

\section{Experimental Verifications}

To verify the effectiveness of the optimum design experimentally, the predicted results of the friction torque and spring coefficient of air film are compared with the measured data for the case of the herring bone grooved bearings. The specifications and the geometries of the test bearings are shown, respectively, in Table 3 and Fig.9, in which the specifications of conventional designed bearing $B$ has the same specifications as the bearing $A$ except the groove depth $h_{g}$. 
Table.3 Specification of bearings

\begin{tabular}{|c|c|c|c|}
\hline & $\begin{array}{c}\text { Optimized } \\
\text { bearing }\end{array}$ & $\begin{array}{c}\text { Conventional } \\
\text { used A }\end{array}$ & $\begin{array}{c}\text { Conventional } \\
\text { used B }\end{array}$ \\
\hline$h_{g}[\mu \mathrm{m}]$ & 30 & 10 & 20 \\
\hline$R_{s}$ & 0.4 & \multicolumn{2}{|c|}{0.76} \\
\hline$\alpha$ & 0.9 & 0.6 \\
\hline$\beta[\mathrm{deg}]$ & 15 & 16 \\
\hline$N$ & 24 & 24.32 \\
\hline$r_{s}[\mathrm{~mm}]$ & 12.8 & \multicolumn{2}{|c|}{24.8 (given) } \\
\hline$r_{1}[\mathrm{~mm}]$ & \multicolumn{3}{|c|}{32 (given) } \\
\hline$r_{2}[\mathrm{~mm}]$ & \multicolumn{3}{|c|}{} \\
\hline
\end{tabular}

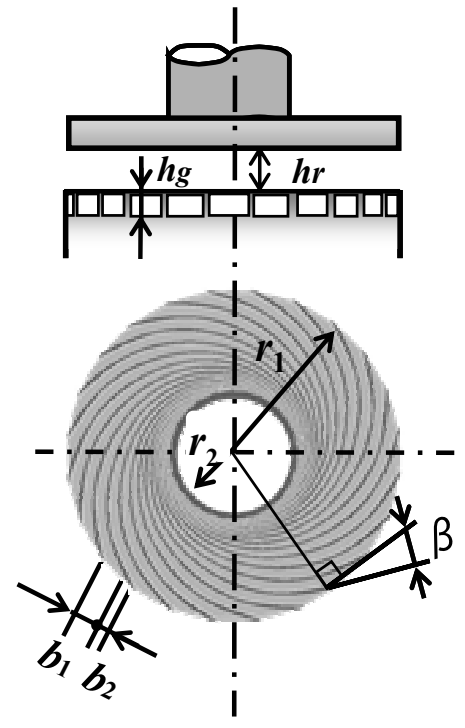

(a) Optimized bearing

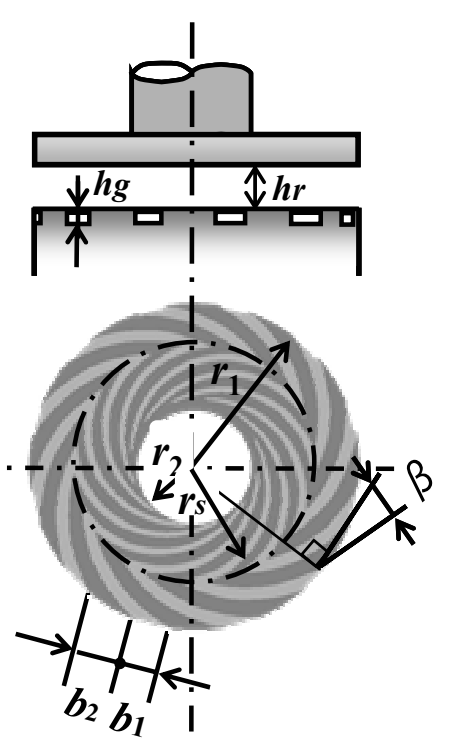

(b) Conventional designed bearing

Fig. 9 Geometry of test bearings

Figure 10 shows the overview of specially designed test rig for the measurements, which is the same test rig as reported in the previous paper ${ }^{(9)}$. The test rig consists of a rotor (1), test bearing(9) and stator(6). The rotor is driven with a built-in type, high frequency motor(2), and both edges of the shaft are supported in the radial direction by ceramic type ball bearings(3). The test bearing is fixed tightly to the stator through the attachment with high parallelism between the runner and bearing surfaces. The stator is supported by the hydrostatic air film bearing(7) in the radial direction, so it is possible to get one-degree of freedom motion of the stator in the axial direction without friction. Moreover, the stator is hang by the micrometer 8 in the axial direction before starting the shaft rotation and the stator is lowered carefully through the micrometer so as to form the complete air film in the bearing gap without contact.

In the experiments, the friction torque on the bearing surface and spring coefficient of air film are measured for rotor speeds from 38000rpm to 42000rpm under the constant mass of $m=1.5 \mathrm{~kg}$. The friction torque on the bearing surface is measured directly through a set of strain gauges attached oppositely at the upper part for the stator. The spring coefficient of air film is obtained by applying experimental modal analysis.

Table 4 and 5 show, respectively, the accuracy of measurements for the averaged data of friction torque and spring coefficient of air film from 38000rpm to 42000rpm. In these tables, the averaged value means the mean value of all measured data for each bearing, and the accuracy means the ratio of standard deviation to the mean value. As can be seen in 


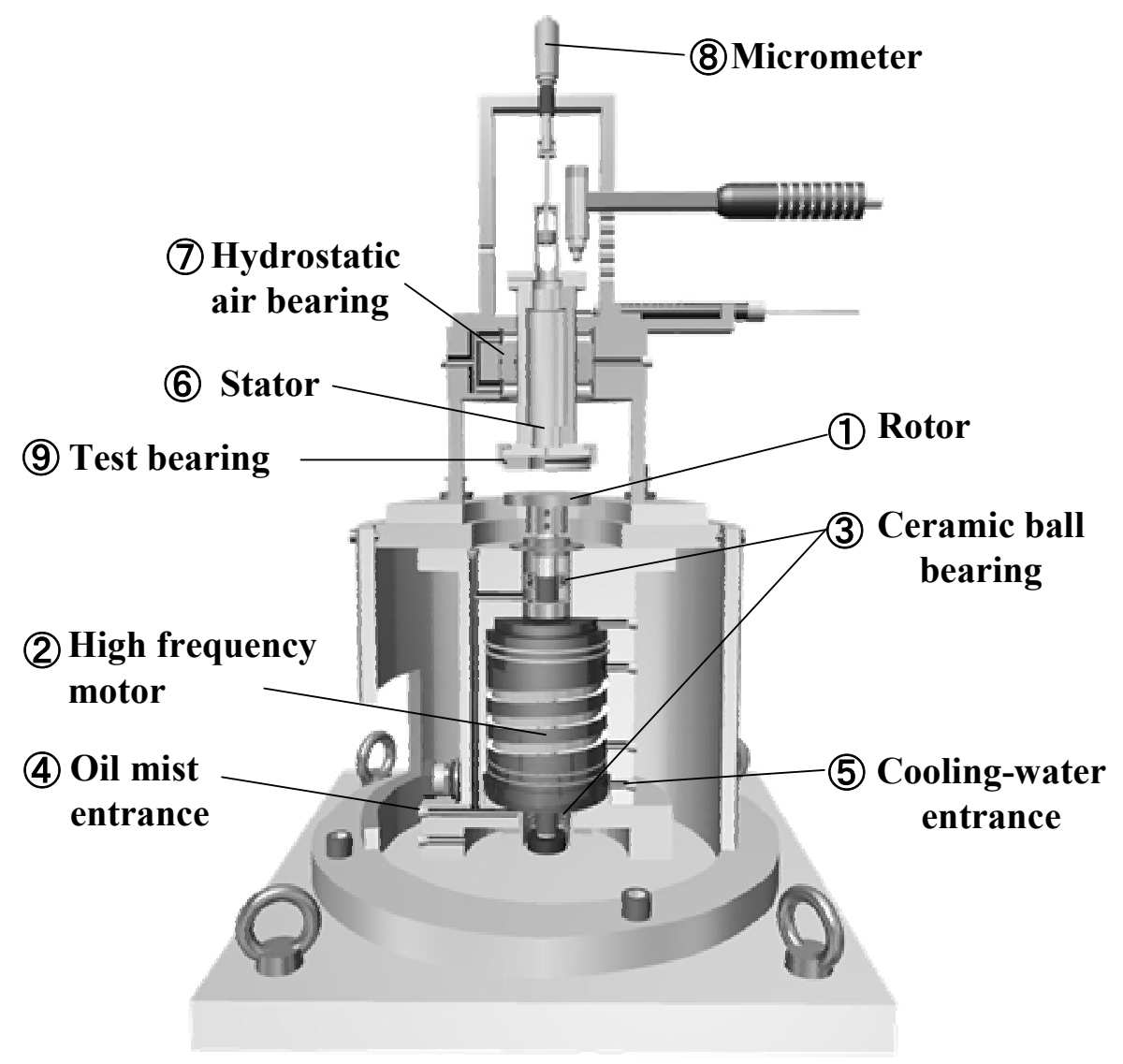

Fig.10 Overview of test rig

Table 4 Measurement accuracy in friction torque

\begin{tabular}{|c|c|c|c|}
\hline & $\begin{array}{c}\text { Averaged value } \\
{[\mathbf{N m}]}\end{array}$ & $\begin{array}{c}\text { Standard deviation } \\
{[\mathbf{N m}]}\end{array}$ & $\begin{array}{c}\text { Accuracy } \\
{[\mathbf{\%}]}\end{array}$ \\
\hline Optimized & $3.99 \times 10^{-3}$ & $12.7 \times 10^{-5}$ & 3.19 \\
\hline Conventional A & $6.09 \times 10^{-3}$ & $6.26 \times 10^{-5}$ & 1.02 \\
\hline Conventional B & $4.60 \times 10^{-3}$ & $8.59 \times 10^{-5}$ & 1.86 \\
\hline
\end{tabular}

Table 5 Measurement accuracy in spring coefficient

\begin{tabular}{|c|c|c|c|}
\hline & $\begin{array}{c}\text { Averaged value } \\
{[\mathbf{N} / \mathbf{m}]}\end{array}$ & $\begin{array}{c}\text { Standard deviation } \\
{[\mathbf{N} / \mathbf{m}]}\end{array}$ & $\begin{array}{c}\text { Accuracy } \\
{[\mathbf{\%}]}\end{array}$ \\
\hline Optimized & $2.81 \times 10^{6}$ & $2.14 \times 10^{4}$ & 0.76 \\
\hline Conventional A & $3.57 \times 10^{6}$ & $3.58 \times 10^{4}$ & 1.00 \\
\hline Conventional B & $2.52 \times 10^{6}$ & $2.36 \times 10^{4}$ & 0.94 \\
\hline
\end{tabular}

these tables, the accuracy of measurements for the friction torque is less than 3.2 percent and the accuracy for the spring coefficient is less than 1.0 percent, respectively,. Therefore, the measurement method is reasonable to measure the friction torque and spring coefficient of air film.

Figures 11 through 13 show the variation of the friction torque, spring coefficient of air film and the objective function with rotational shaft speed for the optimized and conventional designed herring bone grooved bearings. For all bearings, the predicted results agree well with the measured data, and the effectiveness of the optimum design presented in this paper is verified experimentally. 


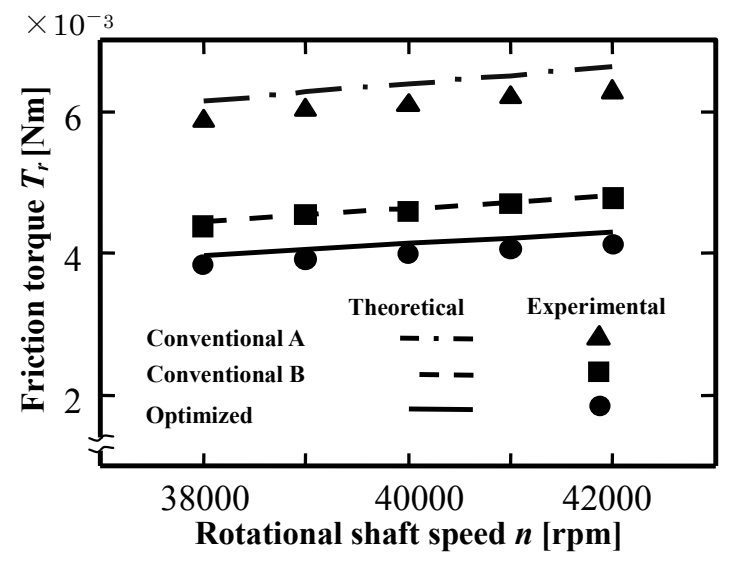

Fig.11 Friction torque with rotational speed

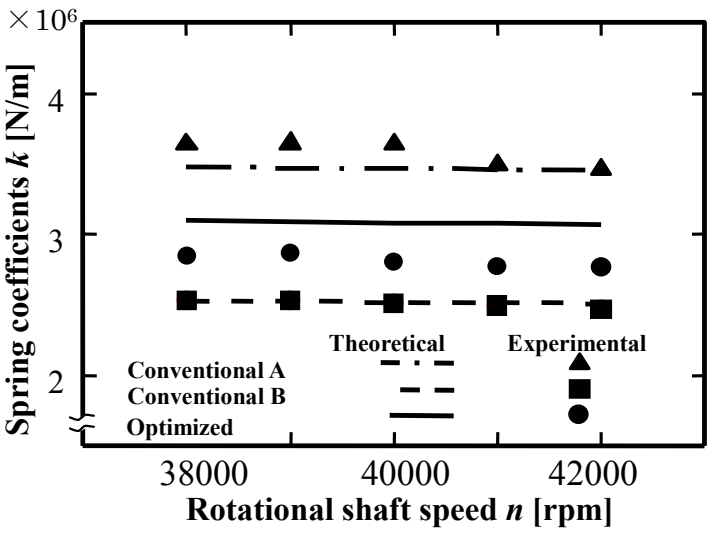

Fig.12 Spring coefficient with rotational speed

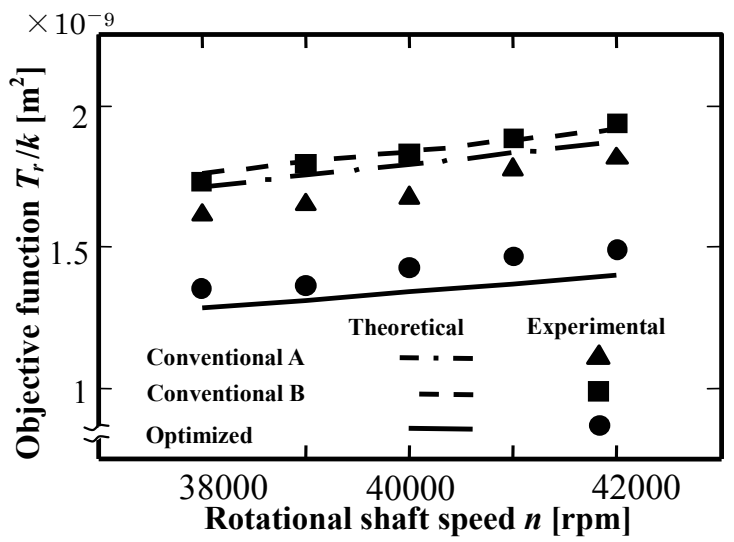

Fig.13 Objective function with rotational speed

\section{Conclusions}

In this paper, the optimum design problems for the spiral and herring bone types of grooved air film thrust bearings are formulated and some examples of optimum design are presented. Moreover, the predicted results are compared with the measured data to verify the effectiveness of optimum design. The conclusions are briefly summarized as follows.

(1) The dimensions of optimized spiral and herring bone types of grooved bearings quietly differ from the conventional designed bearings based on MTI data for the given values of the load applied. However, the optimized geometry of herring bone 
(2) The predicted results of friction torque and spring coefficient of air film based on the present methodology agree well with the measured data, and the effectiveness of optimum design is verified experimentally.

(3) The sensitivity of the rotational shaft speed on the groove geometry of optimized bearings is negligible small.

(4) The value of the objective function, defined as the ratio of friction torque to spring coefficient, is decreased about 25 percent by the optimum design as compared with the case of conventional designed bearings based on MTI data.

\section{References}

(1) Ausman, J. S., Approximate Analytic Solution for Self Acting Gas Lubrication of Stepped Section Thrust Bearing, ASLE Transactions, 81(1959), 135-146.

(2) Castelli, V. and Pirvics, J., Review of Method in Gas Bearing Film Analysis, Transactions of the ASME, Journal of Lubrication Thechnology, 90, 4 (1968), 777-792.

(3) Artiles, A. et al., H. Design Analysis of Rayleigh-Step, Floating Seals, ASLE Transactions, 110, 3(1988), 427-443.

(4) Shapiro, W., et al., Analysis and Design of Gas Lubricated Sectored, Floating Ring Seals, Transactions of the ASME, Journal of Tribology, 27, 4(1984), 321-331.

(5) Malanoski, S. B. and Pan, C. H. T., The Static and Dynamic Characteristics of Spiral-Grooved Thrust Bearings, Transactions of the ASME, Journal of Basic Engineering, 87 (1965), 547-558.

(6) James, D. D. and Potter, A. F., Numerical Analysis of the Gas-Lubricated, Spiral-Groove Thrust Bearing Compressor, Transactions of the ASME, Jouanal of Lubrication Technology, 89 (1967), 439-444.

(7) Lipschity, A., et al., A Bi-Directional Gas Thrust Bearings, STLE Tribology Transactions, 34, 1(1991), 9-16.

(8) Bonneau, D., et al., Finite Element Analysis of Grooved Gas Thrust Bearings and Grooved Gas Face Seals, Transactions of the ASME, Journal of Tribology, 115(1993), 348-354.

(9) Hashimoto, H. and Ochiai, M. , Theoretical Analysis and Optimum Design of High Speed Gas Film Thrust Bearings, JSME Technical Journal, Journal of Advanced Mechanical Design, Systems, and Manufacturing, (Under submitted).

(10)Hashimoto, H. and Hattori, Y., Improvement of the Static and Dynamic Characteristics of Magnetic Head Sliders by Optimum Design, Transactions of the ASME Journal of Tribology, 122-1(2002), 280-287.

(11)RPI-MTI, Design of Gas Bearing(1967). 